\title{
Selected problems of motor vehicle maintenance after side impact collision
}

\author{
Jarosław Zalewski ${ }^{1, *}$ \\ ${ }^{1}$ Warsaw University of Technology, Faculty of Administration and Social Sciences, Pl. Politechniki 1, \\ 00-661 Warsaw, Poland
}

\begin{abstract}
Over the years certain amount of works have been devoted to the selected aspects of motor vehicle collision modeling. Also, in some previous works by the author of this paper the selected problems related to collision modelling have been included. Those papers concerned mostly relations of the obtained crash simulation results to analytical results based on the Routh method, including restitution of the velocities normal and tangential to the plane of crash common for both vehicles involved in the accident. The aim of this paper is to present the selected problems related to the side impact motor vehicle collision modeling, which can be considered as a cause for certain diagnostic problems related to further maintenance of such vehicle after repair.
\end{abstract}

\section{Introduction}

Over the years certain amount of works have been devoted to the selected aspects of motor vehicle collision modeling. Also, in some previous works by the author of this paper the selected problems related to collision modelling have been included. This can be referred e.g. to [4], [5], [7] and [12]. Those papers concerned mostly relations of the obtained crash simulation results to analytical results based on the Routh method, including restitution of the velocities normal and tangential to the plane of crash common for both vehicles involved in the accident. The Routh method has been presented e.g. in [6].

Other more specific problems in road vehicle collision modeling were, for example, influence of the trailer towed by a vehicle [11] or assumption, that the road plane is not flat during collision [11], or even the problem of crashing into a concrete road barrier [9]. Almost all of these problems have been related to the frontal impact of the vehicle.

Not many works however have been devoted to the side impact collision. Some examples of this problem are presented e.g. in [1]. There are two reasons for the small amount of results for side impact. First, it is difficult to specify the direction of the impulse force vector for both vehicles involved. Second, there are not many works showing both experimental and theoretical results concerning the tangential coefficient of restitution, which is necessary to describe the phenomena occurring between the surfaces of the colliding vehicles. Such analyses have been presented e.g. in [3] and [6].

The aim of this paper is to present the selected problems related to the side impact motor vehicle collision modeling, which can be considered as a cause for certain diagnostic problems related to further maintenance of such vehicle after repair.

\section{Selected aspects of road collision modeling}

To properly describe the side impact crash, it seems necessary to make certain assumptions: - quasi-stiff models of the vehicles involved in a collision;

- bodies of the vehicles represented by sets of cuboids of certain mass and stiffness, which do not change during the collision;

\footnotetext{
*Corresponding author: jzalewsk@ans.pw.edu.pl
} 
- the motion of the vehicles is realised on a flat road surface. However, in further research both uneven and sloping road may be considered;

- in more complicated vehicle models nonlinear suspension may be included, but in the presented analysis it will be omitted.

Some additional assumptions may be related to the coordinate systems. For example, following [6], two basic, inertial coordinate systems may be included in the collision model, in which the planar motion is considered. First, marked in fig. 1 as $O x y z$, the inertial global system may be assumed. Second, also shown in fig. 1 the inertial O'ntb system may be adopted as a local axis system, related only to both vehicles, located at the geometric center of the crash (the point of initial contact between the two vehicles).
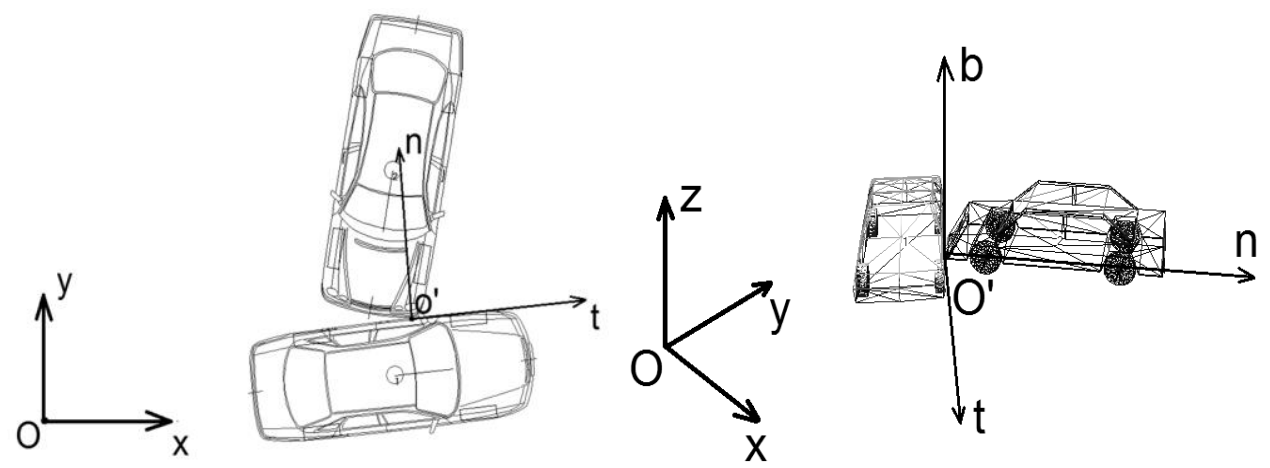

Fig. 1. Example of the possible coordinate systems assumed in the mathematical model of side impact crash between two vehicles [own research based on PC-Crash 8.0].

As for the resultant motion, the proposition of coordinate systems is also presented in fig. 1, where two systems are shown, with vertical axes ( $z$ and $b$ ) included. As for the axes symbols, $t$ marks the tangential direction, $n$ - normal, and $b$ - binormal (normal to the $n-t$ plane).

Additionally, some conditions related to the location of the force impulses in the colliding vehicles may be considered:

- in the planar motion both impulse components may be located along the tangential $(t)$ and the normal ( $n$ ) direction (fig. 1). The returns of these vectors should be opposite for each vehicle. i.e. if for one vehicle the vectors of normal and tangential impulse component point along the $n$ and $t$ axes respectively, then for the other vehicle they should point in the opposite direction;

- in the resultant motion the vertical component of crash impulse may be located along the binormal axis $(b)$, with the return of its vector pointing upwards in accordance with the return of the $O^{\prime} b$ axis, for both vehicles, or, like in case of normal and tangential, in the opposite directions.

Such parameters, as wheel camber angles or the vehicle body tilt in relation to the vertical axis can be omitted for simpler cases but may be included in the more complicated mathematical model of the crash.

It is important to remember, that the main goal of collision modeling is to enable calculation of the transverse and rotational velocities for both vehicles at the end of the crash, which lasts for a very short period of time. It means that in certain situations only the basic mathematical formulas are sufficient to do it. However, when there is a necessity to consider many parameters which would make it possible to fully and thoroughly reconstruct or simulate the specific road accident, then the obtained mathematical model may be more complicated and more difficult to solve, especially when the side impact crash is discussed.

\section{Selected problems in side impact collision modeling}


When analysing the side impact crash, two cases have been considered:

- both vehicles realise the planar motion during the impact;

- both vehicles realise the resultant motion during the impact, where the impulses of inertia, centrifugal and transportation forces have been considered.

It has also been assumed that for the description of motion for both vehicles a local coordinate system will be used, which in fig. 1 marked as $O$ 'ntb and its origin is placed in the point of initial contact of both vehicles. For the planar motion only normal $(n)$ ant tangential $(t)$ axes have been used, while for the resultant motion also so called binormal $(b)$ axis has been considered.

\subsection{Side impact model with vehicles realising planar motion}

In fig. 2 the impulses of the impact force are presented with respect to both the planar (left part of the figure) and the resultant motion (right part). Let us accept that, regarding the difficulty in specifying the direction of the crash impulse vector, we can divide it into two components for planar, and three for the resultant motion, for each vehicle. Let the impulses be market with $S$ and the lower index means tangential, normal and binormal as in the marking of the axes in the adopted coordinate system. Hence the normal impulse will be directed along the normal axis $n$, the tangential components will be located along the $t$ axis and the binormal one - along the $b$ axis. However, for the considered example the binormal component of the crash impulse will be returned in the same direction for both vehicles.

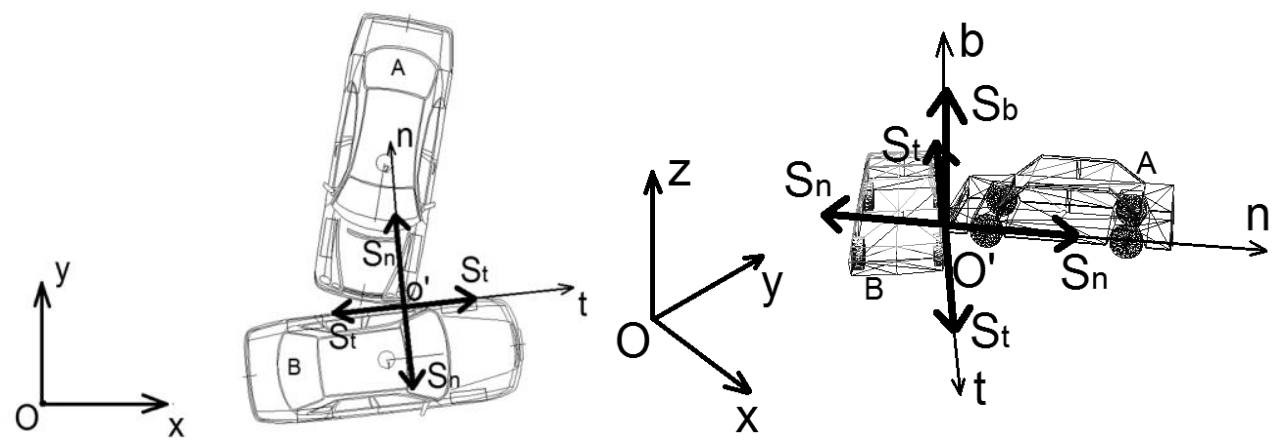

Fig. 2. Example of the possible location of the impact force impulse components for planar and resultant motion during the collision [own research based on PC-Crash 8.0].

\subsection{Side impact model with vehicles realising resultant motion}

For the resultant motion the adopted description will be the same as for the planar case (fig. 2). However, the consequences of such phenomena, which allows a momentary loss of contact between at least one wheel and the road surface for each vehicle, may be different than for the planar motion. It is not the intention of the author to examine the consequences so thoroughly, that they will be divided into these two cases (planar and resultant motion). The main goal of this part is to present the differences in the attempt towards collision modeling. Adopting a mathematical model that would describe the resultant motion during the impact seems to be more exact when the broader scope of phenomena is to be examined, because it offers more realistic attempt e.g. in accident reconstruction or collision analysis.

The scheme of the mutual location of both vehicles involved in a side impact crash with the impulses generated during resultant motion is presented in fig. 3 . 


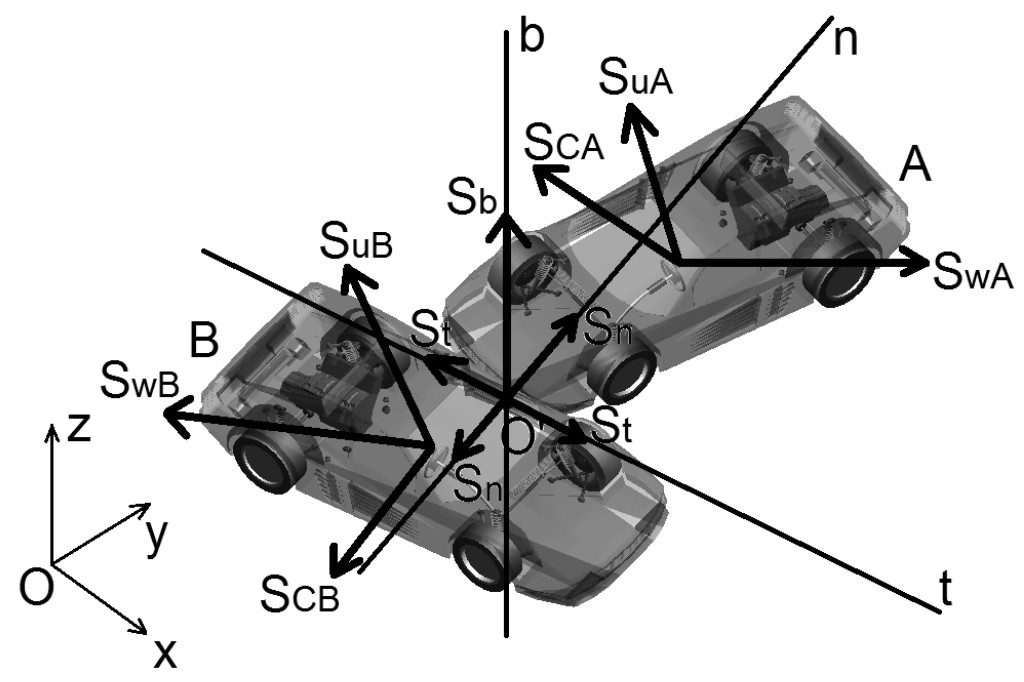

Fig. 3. The scheme of a mutual location of both vehicles with impulses occurring in resultant motion [own research based on MSC Adams/Car].

In fig. 3 certain parameters have been marked for vehicle $A$ crashing into the side part of a vehicle $B$. Apart from the previously discussed impulses located in the geometric center of the impact, additional impulses characteristic for the resultant motion have been assumed. $S_{w A}$ means the impulse of the inertial force of vehicle $A$ relative to the point $O$ ', $S_{u A}$ marks the impulse of the transportation force of a vehicle $A$, while $S_{C a}$ is the impulse of the Coriolis force of a vehicle $A$. The same vectors have been assumed for the vehicle $B$, only the markings in the lower index should refer to the vehicle $B$.

More thorough analysis of these impulses for the front impact has been presented, among others in [5] and [7], where attention has been paid to their location relative to the $O$ 'ntb axis system (like in fig. 3). Like in the given example, the vectors of these impulses have been attached to the centers of mass of both vehicles.

Other attempt towards vehicle crash modeling allows assuming the additional factors, such as momentary vehicle oversteer, road conditions or mass distribution in a colliding vehicle, which can generate additional impulses attached not necessarily to the center of mass of a given vehicle. Hence the process of vehicle collision may be more complicated than previously imagined, especially from the point of view of mathematical modeling. Such attempt has been previously considered e.g. in [7] and [10].

In further part certain approach to the consequences of a side impact crash have been discussed.

\section{General mathematical model for the side impact crash between two vehicles}

In general, the vehicle side impact crash model can be presented as a set of equations (1) related to the scheme presented in fig. 3. The apostrophe in the upper index marks the value after the collision. Note, that by the collision duration should be understood as a period of contact between two vehicles, which corresponds to the sudden decrease or increase in velocity at a little time step. 


$$
\begin{aligned}
& m_{A}\left(v_{A n}^{\prime}-v_{A i n}\right)=\sum_{i=1}^{k} S_{n A}+\sum_{i=1}^{k} S_{i w n A}+\sum_{i=1}^{k} S_{i u n A}+\sum_{i=1}^{k} S_{i C n A} \\
& m_{A}\left(v_{A t}^{\prime}-v_{A t}\right)=\sum_{i=1}^{k} S_{t A}+\sum_{i=1}^{k} S_{i w t A}+\sum_{i=1}^{k} S_{i u t A}+\sum_{i=1}^{k} S_{i C t A} \\
& m_{A}\left(v_{A b}^{\prime}-v_{A b}\right)=\sum_{i=1}^{k} S_{b A}+\sum_{i=1}^{k} S_{i w b A}+\sum_{i=1}^{k} S_{i u b A}+\sum_{i=1}^{k} S_{i C b A} \\
& I_{A}\left(\omega_{A}^{\prime}-\omega_{A}\right)=\sum_{i=1}^{k} S_{t A} n_{i A}+\sum_{i=1}^{k} S_{n A} t_{i A}+\sum_{i=1}^{k} S_{b A} b_{i A} \\
& m_{B}\left(v_{B n}^{\prime}-v_{B i n}\right)=\sum_{i=1}^{k} S_{n B}+\sum_{i=1}^{k} S_{i w n B}+\sum_{i=1}^{k} S_{i u n B}+\sum_{i=1}^{k} S_{i C n B} \\
& m_{B}\left(v_{B t}^{\prime}-v_{B t}\right)=\sum_{i=1}^{k} S_{t B}+\sum_{i=1}^{k} S_{i w t B}+\sum_{i=1}^{k} S_{i u t B}+\sum_{i=1}^{k} S_{i C t B} \\
& m_{B}\left(v_{B b}^{\prime}-v_{B b}\right)=\sum_{i=1}^{k} S_{b B}+\sum_{i=1}^{k} S_{i w b B}+\sum_{i=1}^{k} S_{i u b B}+\sum_{i=1}^{k} S_{i C b B} \\
& I_{B}\left(\omega_{B}^{\prime}-\omega_{B}\right)=\sum_{i=1}^{k} S_{t B} n_{i B}+\sum_{i=1}^{k} S_{n B} t_{i B}+\sum_{i=1}^{k} S_{b B} b_{i B} \\
& i=1, \ldots, k
\end{aligned}
$$

where:

- $m_{A}, m_{B}$ - mass of the vehicle $A$ and $B$ respectively;

- $v_{A t}, v_{B t}-$ a component of vehicle velocity ( $A$ and $B$ respectively) tangential to the common plane of contact during collision;

- $v_{A n}, v_{B n}$ - a component of vehicle velocity ( $A$ and $B$ respectively) normal to the common plane of contact during collision;

- $v_{A b}, v_{B b}$ - a component of vehicle velocity ( $A$ and $B$ respectively) binormal to the plane of motion (road surface) during collision;

- $\omega_{A}, \omega_{B}$ - angular velocity of a vehicle $A$ and $B$ respectively;

- $I_{A}, I_{B}-$ moment of inertia relative to the vertical axes passing through the center of mass of each vehicle;

- $S_{n A}, S_{t A}, S_{n B}, S_{t B}, S_{b A}, S_{b B}$ - normal, tangential and binormal components of the impulse of the impact force for the vehicle $A$ and $B$ respectively;

- $S_{i w n}, S_{i w t}, S_{i w b}$ - normal, tangential and binormal components of a force resulting from the inertial force of vehicle $A$ and $B$ respectively, relatively to the point $O^{\prime}$ (geometric center of the impact);

- Siun, $S_{i u t}, S_{i u b}$ - normal, tangential and binormal components of a transportation force of vehicle $A$ and $B$, resulting from the motion of $O^{\prime}$ (geometric center of the impact) versus the origin of a global axis system $O x y z$ (fig. 3);

- $S_{i C n}, S_{i C t}, S_{i C b}$ - normal, tangential and binormal components of a Coriolis force of a vehicle $A$ and $B$, assumed in the resultant motion of those vehicles during the impact;

- $n_{i A}, t_{i A}, b_{i A}, n_{i B}, t_{i B}, b_{i B},-$ coordinates of the centers of mass of both vehicles $(A$ and $B)$ in relation to the point $\mathrm{O}^{\prime}$ (geometric center of the impact) according to the direction of the axes $O^{\prime} n, O^{\prime} t$ oraz $O^{\prime} b$ (fig. 3).

The main goal of each vehicle collision model is to derive a kinematic state of before or after the impact. The kinematic state, allowing calculation of the velocities after the crash, has been presented among others in [4] and [6].

For both planar and resultant motion it is necessary to be able to define the values of impulses both for the impact force and the resultant motion. It is also important to know the values of the necessary coefficient of restitution used to define these impulses, which has been the subject of such works as for example [5] and [8].

Road vehicle collision modeling can be useful in understanding the process of vehicle collision and the possible influence of certain factors on the results. However, the other attempt may provide understanding the possible consequences of vehicle collision, especially 
side impact, on the diagnostic aspects in the process of post-accident vehicle repair and allowance for further maintenance.

\section{Possible considerations on the phenomena occurring in contact area during the side crash}

One of the most important parameters in vehicle crash modeling is the coefficient of restitution. As for the front impact collision it may seem that the phenomena occurring in the area of contact between the vehicles are of less importance due to the fact, that the vehicles involved in such collision penetrate each other and the deformations are mainly related to the damage of certain, crashworthy and energy absorbing structures of the front or the back part of a vehicle. Hence the only coefficient of restitution often taken into account is the coefficient related to the normal velocities, i.e. the components of velocity parallel to the direction of motion of a given vehicle.

Quite differently is for the side impact crash, where deformations can be immediately transferred to the parts of a vehicle body which do not absorb the kinetic energy of a collision. If those deformations reach the passenger compartment, then people being in such vehicle are posed to the risk of health or life loss. In side impact collisions modeling two coefficients of restitution should be taken into account: the one for normal (here called $R_{n}$ ) and the other for tangential (here called $R_{t}$ ) components of velocity. In resultant motion tangential coefficient of restitution should be considered, e.g for $O^{\prime} t$ and $O^{\prime} b$ directions in fig. 3 .

As for the direction of the impulse of impact force between two colliding vehicles, certain assumptions have been made in [6], where the first part of the following hypothesis, concerning the planar motion during a crash, has been adopted. However, the second part of this hypothesis has not been previously published. Note, that these simple calculations are only the example to simplify selected aspects of the more than usual complicated vehicle crash mathematical model.

If the angle between the colliding vehicles is close to 90 degrees, then the component of the impulse in direction $O^{\prime} t$ can be omitted, because, given that the impulse can be decomposed into components along the three axes of the $O$ 'ntb system (fig. 3), the sine of this angle close to 90 degrees will be close to zero.

If, however, the angle between the colliding vehicle ( $A$ in fig. 3 ) and the plane of the mutual contact will not be close to 90 degrees, then its sine will not produce a value close to zero, and hence it may not be omitted in the equations describing the kinematic state during the crash.

In relation to the considerations, provided that the specific example of side impact crash is discussed, certain formula describing relations between the normal and tangential component of the impact force impulse has been presented, according to fig. 4:

where:

$$
S=\sqrt{S_{n}^{2}+S_{t}^{2}}, S_{n}=S \cos \alpha, S_{t}=S \sin \alpha
$$

- $S$ - impulse of the impact force;

- $S_{n}$ - the normal component of the impact force impulse along the $O$ 'n axis (fig. 4);

- $S_{t}$ - the tangential component of the impact force impulse along the $O^{\prime} t$ axis (fig. 4);

$-\alpha$ - the angle between the colliding vehicle and the perpendicular to the plane of a common contact (fig 5).

It should be mentioned that for the small values of $\alpha$ :

$$
S_{n}=S \cdot \cos \alpha=S \text { and } S_{t}=S \cdot \sin \alpha=S \cdot \alpha \approx 0
$$




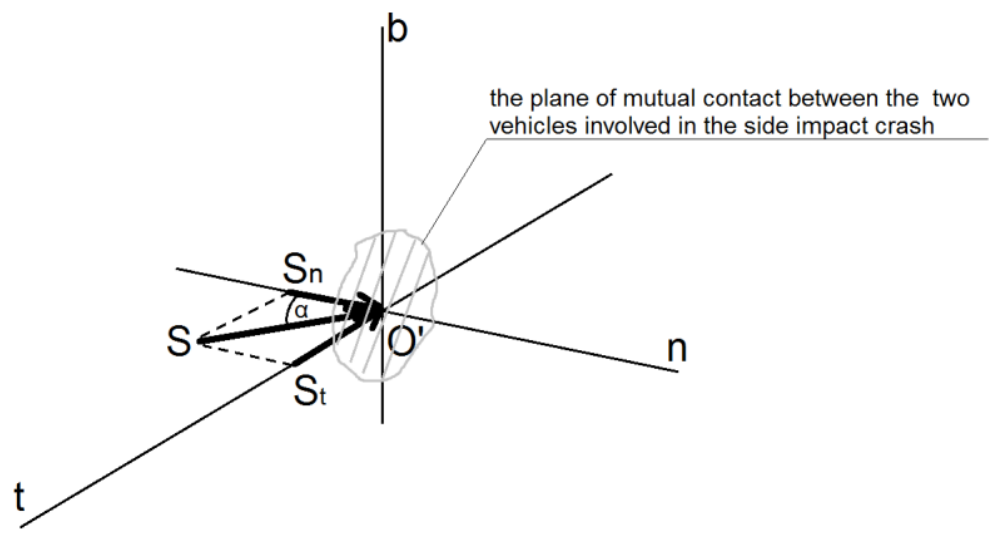

Fig. 4. Components of the impulse of impact force in planar motion side crash [own research]

As for the binormal component of the impact force impulse, its presence cannot be described in such simple manner as for the tangential direction, because the phenomena may change during the collision period, provided the resultant motion is taken into account and some wheels of at least one vehicle involved in the collision will lose contact with the ground.

However, adopting the same assumptions given above, in general the binormal component in case of resultant motion during the crash can be defined using another angle. Let $\beta$ be the angle between the n-t plane and a vector of the impact force impulse. Then, according to fig. 5 all three components of the crash impulse will be:

where:

$$
S=\sqrt{S_{n}^{2}+S_{t}^{2}+S_{b}^{2}}, S_{n}=S \sin \beta \cos \alpha, S_{t}=S \sin \beta \sin \alpha, S_{b}=S \cos \beta
$$

- $S_{b}$ - the binormal component of the impact force impulse along the $O^{\prime} t$ axis (fig. 5);

- $\beta$ - the angle between the colliding vehicle and the perpendicular to the road (fig 5).

It also should be mentioned that for the small values of $\beta$ :

while for the large values of $\beta$ :

$$
S_{n}=S \cdot 0 \cdot \cos \alpha \approx 0, S_{t}=S \cdot 0 \cdot \sin \alpha \approx 0, S_{b}=S \cdot \cos \beta \approx S,
$$

$$
S_{n}=S \cdot \sin \beta \cos \alpha, S_{t}=S \cdot \sin \beta \sin \alpha, S_{b}=S \cdot \cos \beta \neq 0
$$

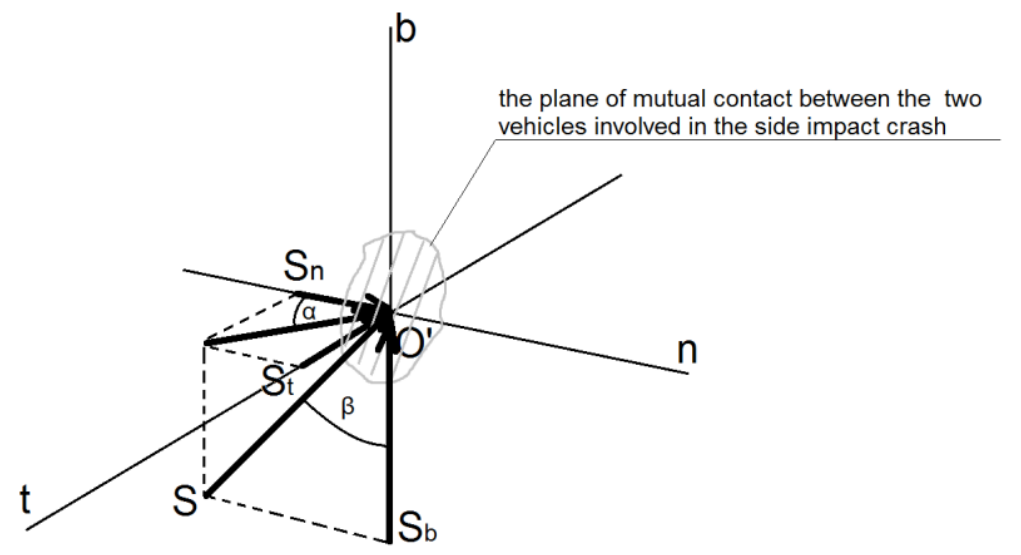

Fig. 5. Components of the impulse of impact force in planar motion side crash [own research]

If, however, the angle $\beta$ was adopted between horizontal direction and the impulse $S$, then the components $S_{n}, S_{t}$ and $S_{b}$ would be calculated differently.

\section{Possible consequences of the side impact collisions}


As a side impact crash seems to be one of the most dangerous incidents in road traffic, it is necessary to consider the possible consequences in the aspect of a vehicle construction and maintenance, especially after post-accident repair.

The main result of a side impact crash, depending on the point of application of the impact force impulse, may be different disturbances in geometry of any given motor vehicle body. If the deformations would cover the lower parts of a body, i.e. the floor board, which is made of the closed, empty inside, omega-like profiles, then this qualitative assessment would be a possible factor compelling the given vehicle to be checked towards the location of the specific points in its body. However, there is a question whether these specific points, whose location informs only about a proper geometry, is sufficient enough to be used as a criterion allowing the given vehicle, repaired after accident, for further maintenance.

It seems obvious that the certain, specified criteria is necessary to be able to assess the vehicle involved in a collision to be either repaired, or utilisation.

\section{References}

1. Abe M., Morisawa M., Arai K. Sato T. B., Three-dimensional Behavior of Vehicle at Oblique Side Collision - Computer Simulation Using Dynamical Model, JSAE, 1995.

2. Brach R. M., Restitution in Point Collisions, Contribution to Computational Aspect of Contact, Impact and Penetration, Ronald F. Kulak and Leonard E. Schwer, Editors, Elmepress International, Lausanne, Switzerland, 1991.

3. Ishikawa H., Impact model for accident reconstruction - normal and tangential restitution coefficients. SAE paper 930654, 1993.

4. Kisilowski J., Zalewski J., Analysis of Chosen Aspects of a Two-Car Crash Simulation, Telematics in the Transport Environment, Communications in Computer and Information Science 329, Springer, 2012.

5. Kisilowski J., Zalewski J., Analysis of the Selected Aspects of a Crash Between Two Vehicles, Advances in Intelligent Systems Research, Atlantis Press, 2013.

6. Kisilowski J., Zalewski J, Modelowanie zdarzeń w ruchu drogowym, Wydawnictwo Instytutu Technologii Eksploatacji, Radom - Warszawa 2016. (in Polish)

7. Kisilowski J., Zalewski J., Wybrane aspekty analizy i modelowania zderzenia samochodów, Technika Transportu Szynowego, 10/2013. (in Polish)

8. Wicher J., O współczynniku restytucji w zderzeniach samochodów, On the coefficient of restitution in the vehicle collisions, Zeszyty Naukowe Politechniki Świętokrzyskiej. Mechanika, 2006. (in Polish)

9. Wicher J., Rekonstrukcja zderzenia samochodu z drogową barierą betonową, Zeszyty Naukowe Instytutu Pojazdów, 2(57)/2005. (in Polish)

10. Wicher J., Stawicki R., Modelowanie zderzenia samochodów, Zeszyty Naukowe Instytutu Pojazdów, 1(31)/99. (in Polish)

11. Wicher J., Sztenke M., Oddziaływanie przyczepy i nachylenia drogi na parametry ruchu podczas zderzenia samochodów, Zeszyty Naukowe Instytutu Pojazdów, 3(29)/98. (in Polish)

12. Zalewski J., Analysis of a road accident in the aspect of mechanics of a frontal crash between two vehicles, Diagnostyka, vol 16 (4), 2015. 